\title{
Efficacy of Preemptive Analgesics and the need to Standardize Anesthetic and Analgesic Management
}

\section{TO THE Editor:}

We read with great interest the article of Peng et al (1) in a recent issue of the journal where the authors performed a randomized, double-blind, placebo-controlled study on 94 patients undergoing total hip arthroplasty and concluded that a single dose of parecoxib $30 \mathrm{~min}$ utes before incision did not provide effective preemptive analgesia for the management of postoperative pain after primary unilateral THA. The authors should be commended for performing a well-designed trial in an important topic (e.g., acute pain) in patients undergoing orthopedic surgery $(2,3)$. The current emphasis on the need to improve overall postoperative recovery and patient satisfaction makes the topic very important in perioperative medicine $(4,5)$.

Although the study of Peng et al (1) was well conducted, there are some questions that could to be clarified by the authors that could be of benefit to the readers. First, the authors did not control for the use of intraoperative analgesics or anesthetics which are known to affect postoperative pain and subsequently recovery. Second, the protocol did not allow for a standard postoperative analgesic control and patients received pethidine and tramadol in a non-standardized fashion. Finally, it is unclear how the authors collected the data and evaluated postoperative pain. It is well known that the use of nurses' evaluation using chart review is not a reliable method (6).

We would welcome comments by the authors as this would help to further clarify the findings of this important clinical trial.

\author{
James Park, MD \\ Department of Anesthesiology \\ Rhode Island Hospital \\ Warren Alpert Medical School of Brown University, \\ Providence, RI \\ Mark C. Kendall, MD \\ Director of Clinical Research \\ Department of Anesthesiology \\ Warren Alpert Medical School of Brown University \\ 593 Eddy Street \\ Providence, RI 02903 \\ E-mail: mark.kendall@lifespan.org
}

\section{References}

1. Peng HM, Wang LC, Wang W, Tang QH, Qian WW, Lin J, Jin J, Feng B, Yin XH, Weng XS, Zhou YX. Preemptive analgesia with parecoxib in total hip arthroplasty: A randomized, double-blind, placebo-controlled trial. Pain Physician 2018; 21:483-488.

2. Chen $Z$, Tang $R$, Zhang $R$, Jiang $Y$, Liu $Y$. Effects of dexmedetomidine administered for postoperative analgesia on sleep quality in patients undergoing abdominal hysterectomy. J Clin Anesth
2017; 36:118-122.

3. Scholz A, Bothmer J, Kok M, Hoschen K, Daniels S. Cebranopadol: A novel, firstin-class, strong analgesic: Results from a randomized phase iia clinical trial in postoperative acute pain. Pain Physician 2018; 21:E193-E206.

4. Burkle CM, Olsen DA, Sviggum HP, Jacob AK. Parturient recall of neuraxial analgesia risks: Impact of labor pain vs no labor pain. J Clin Anesth 2017; 36:158-163.

5. Shick V, Lebovitz EE, Conrad E. The ben- efits of ultrasound-guided continuous sensory nerve blockade in the setting of burn injury: A case report of bilateral continuous superficial peroneal nerve blockade in a patient with severe sleep apnea. J Clin Anesth 2017; 36:62-66.

6. Wanderer JP, Shi Y, Schildcrout JS, Ehrenfeld JM, Epstein RH. Supervising anesthesiologists cannot be effectively compared according to their patients' postanesthesia care unit admission pain scores. Anesth Analg 2015; 120:923-32. 